\title{
The Preventive Effect of Endovascular Treatment for Recurrent Hemorrhage in Patients with Spinal Cord Arteriovenous Malformations
}

\author{
Y. Niimi, H. Matsukawa, DN. Uchiyama, and A. Berenstein
}

\begin{abstract}
BACKGROUND AND PURPOSE: Spinal cord AVMs represent rare and insufficiently studied pathologic entities. Embolization is thought to play an important role in the management of spinal cord AVMs. Factors for recurrent hemorrhage and the impact of endovascular treatment on prevention of recurrent hemorrhage remain to be confirmed. We aimed to assess recurrent hemorrhagic incidence of spinal cord AVMs and its prevention by endovascular treatment.
\end{abstract}

MATERIALS AND METHODS: We reviewed 80 patients with spinal cord AVMs by spinal cord angiography who had hemorrhage before the first endovascular treatment at New York University Medical Center, Beth Israel Medical Center, or Roosevelt Hospital in New York. We compared the baseline and radiologic characteristics of patients with and without recurrent hemorrhage by the log-rank test and the Cox proportional hazards model.

RESULTS: We observed recurrent hemorrhage in 35 (44\%) patients (1/41 patients with endovascular treatment and $34 / 39$ patients without endovascular treatment). The median length of total follow-up was 659 days (interquartile range, 129-2640 days), and the median length from first-to-recurrent hemorrhage was 369 days (interquartile range, 30-1596 days). The log-rank test revealed that endovascular treatment and venous thrombosis reduced recurrent hemorrhage, and associated aneurysm was related to recurrent hemorrhage. Even in multivariate analysis, the endovascular treatment reduced (hazard ratio, 0.027; $P<.0001$ ) and associated aneurysm increased (hazard ratio, $3.4 ; P=.044)$ the risk of recurrent hemorrhage.

CONCLUSIONS: Endovascular embolization is the first choice of treatment for spinal cord AVMs and is effective in preventing recurrent hemorrhage.

ABBREVIATIONS: $\mathrm{ASA}=$ anterior spinal artery; $\mathrm{NBCA}=n$-butyl 2-cyanoacrylate; $\mathrm{SCAVM}=$ spinal cord AVM

$S^{n}$ pinal cord AVMs (SCAVMs) represent rare and insufficiently studied pathologic entities characterized by considerable variation. ${ }^{1}$ Insufficient study of this disease is associated with the rarity and complexity of its diagnosis. ${ }^{2,3}$ Spinal cord arteriovenous fistula is a direct communication between arteries and veins, while spinal cord arteriovenous malformation in its narrow denotation has a nidus, an abnormal vascular network interposed between arteries and veins. On some occasions, both AVF and AVM constitute a shunt, which is also called AVM in its broader

Received July 31, 2014; accepted after revision February 4, 2015.

From the Departments of Neuroendovascular Therapy (Y.N.) and Neurosurgery (H.M.), St. Luke's International Hospital, Tokyo, Japan; Department of Neurosurgery (N.U.), Kanazawa University, Kanazawa, Japan; and Center for Endovascular Surgery (A.B.), Roosevelt Hospital, New York, New York.

Please address correspondence to Yasunari Niimi, MD, Department of Neuroendovascular Therapy, St. Luke's International Hospital, 9-1 Akashi-cho, Chuo-ku, Tokyo 104-8560, Japan; e-mail address: yasunm@luke.ac.jp

http://dx.doi.org/10.3174/ajnr.A4396 meaning. ${ }^{4}$ Spinal cord AVFs and AVMs manifest with sudden or gradual deterioration of sensorimotor function in the extremities and/or of micturition, defecation, or sexual function due mainly to venous hypertension/ischemia and, in some cases, arterial steel, hemorrhage, or mass effect. ${ }^{4}$

There has been advancement in endovascular treatment of SCAVM, including monitoring systems. Treatment of an SCAVM aims to decrease the risk of hemorrhage and arrest the progression of neurologic deterioration. ${ }^{5}$ Embolization is thought to play an important role in the management of SCAVMs, both as a primary treatment and as an adjunct to surgical excision. However, complete cure of SCAVMs by endovascular embolization is exceptional except for spinal cord AVFs, and endovascular treatment often results in partial obliteration of the lesion by target embolization. Target embolization aims at occluding dangerous structures causing hemorrhage or neurologic symptoms, such as aneurysms, high-flow fistulas, and nerve root lesions for radicular pain. To date, various studies investigated the factors related to 


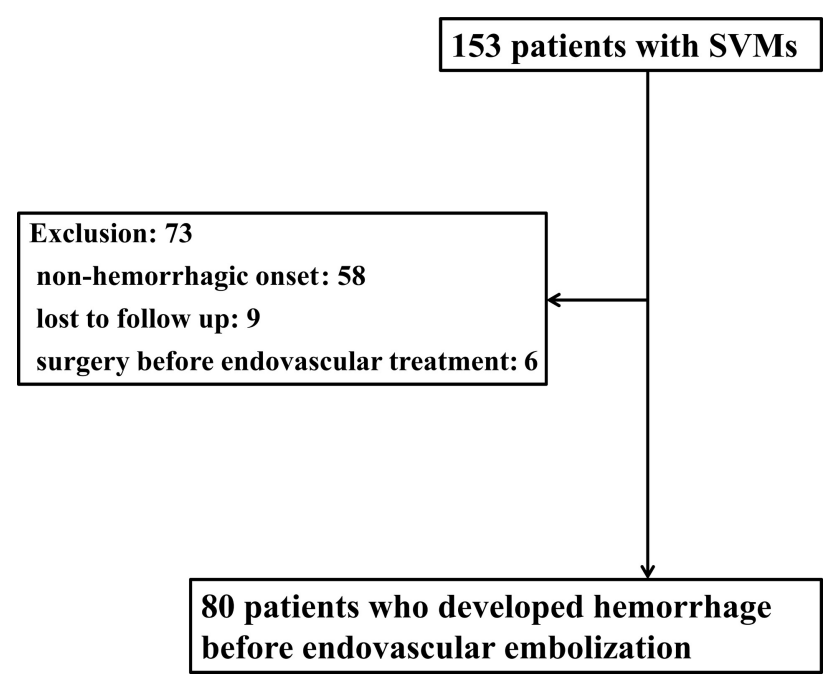

FIG 1. Exclusion criteria of the present study. SVM indicates spinal vascular malformation.

initial hemorrhage. ${ }^{6-10}$ However, factors for recurrent hemorrhage and the impact of endovascular treatment on prevention of recurrent hemorrhage remain to be confirmed. The purpose of this study was to assess the recurrent hemorrhagic incidence of SCAVMs and its prevention by endovascular treatment.

\section{MATERIALS AND METHODS}

The study is reported on the basis of criteria from the Strengthening the Reporting of Observational Study in Epidemiology statement. ${ }^{11}$

\section{Study Participants}

We retrospectively reviewed 153 patients diagnosed with SCAVM by spinal cord angiography at centers where the senior author worked (New York University Medical Center, Beth Israel Medical Center, or Roosevelt Hospital in New York) between April 1980 and October 2010. Exclusion criteria are shown in Fig 1. After exclusions, we analyzed 80 patients with SCAVMs who had hemorrhage before the first endovascular treatment.

\section{Clinical Characteristics}

We collected the following data: age, sex, the presence of SCAVMrelated syndrome, endovascular treatment after the first hemorrhage or radiation therapy, and radiologic characteristics. We defined pediatrics as younger than 18 years of age.

Radiologic characteristics included hemorrhage type, detailed diagnosis, lesion level, the presence of an associated aneurysm, venous thrombosis, venous stricture, venous ectasia, and venous hypertension.

\section{Improvement of Angiographic and Embolization Techniques}

There has been improvement in angiographic and embolization techniques during the 30 years of the study and continuous improvement in knowledge of disease, angiographic imaging quality, endovascular equipment including microcatheters, microguidewires, embolic agents, and monitoring systems. We started by using guidewire-assisted and flow-guided microcatheters in the late 1980s. As a monitoring system, somatosensory evoked potentials introduced in the mid-1980s, and transcranial motor evoked potentials, in the mid-1990s as well as the provocative testing method. ${ }^{12}$ We switched from isobutyl cyanoacrylate to $n$-butyl 2-cyanoacrylate (NBCA) as a liquid embolic agent in the late 1980s. Detachable coils became available for certain diseases in the early 1990s. All these improvements enhanced the safety and adequacy of endovascular occlusion of SCAVMs. However, our conceptual approach for endovascular treatment of an SCAVM (ie, partial targeted embolization) is, in principle, unchanged during the 30 years of the study because we still have difficulty in completely obliterating an SCAVM by embolization itself. Therefore, we decided to analyze all cases of SCAVMs during 30 years.

\section{Current Angiographic/Embolization Protocol}

All angiographic procedures were performed with the patients under general anesthesia with neuroleptic agents and neurophysiologic monitoring, including somatosensory evoked potentials and transcranial motor evoked potentials. Superselective catheterization of the appropriate feeding pedicles was performed by using a microcatheter before embolization to analyze the vascular anatomy of the lesion. Pharmacologic provocative testing was performed with superselective injection of amobarbital sodium and lidocaine when indicated. The details of the indication, technique, and results of neurophysiologic monitoring and provocative testing for SCAVMs have been described previously. ${ }^{12}$ After hemorrhage with neurologic deficits, patients were treated conservatively until their neurologic deficits became stabilized, and we then performed endovascular treatment if indicated. We perform targeted partial embolization for nidus-type SCAVMs to close aneurysms and fistulas to prevent hemorrhage and improve spinal cord hemodynamics to treat symptomatic myelopathy/venous hypertension. Complete angiographic cure is not the goal with most intradural lesions with the exception of AVFs. $n$-butyl 2-cyanoacrylate was the main embolic agent for both intra- and extradural lesions. Particulate embolization was used when NBCA embolization was not feasible on the basis of the flow dynamics of the lesion. Coils were used for embolization of aneurysms, high-flow fistulas, and protection of the normal vascular territories when embolizations were performed from proximal positions (nonintranidal). Ethylene-vinyl alcohol copolymer (Onyx; Covidien, Irvine, California) was not used for intradural lesions. The embolization procedure was repeated in a staged fashion if considered preferable.

\section{Outcome}

The treatment results were confirmed by angiography after embolization. MR imaging was also performed if indicated. Clinical follow-up was performed in a multidisciplinary clinic attended by both interventional neuroradiologists and neurosurgeons. In case of any clinical suspicion of recurrence or progression of the disease, MR imaging or angiography or both were repeated at that time. Repeat embolization was performed on the basis of the angiographic findings. Annual follow-up MR imaging was performed if the patient was neurologically stable. If there was any change in the appearance, spinal angiography was performed with the intent to treat. 
Table 1: Duration between initial and recurrent hemorrhage in patients with SCAVMs

\begin{tabular}{lcc}
\multicolumn{1}{c}{ Duration } & No. $(\boldsymbol{n}=\mathbf{3 5})$ & Percentage \\
\hline <7 Days & 6 & 18 \\
8-31 Days & 5 & 14 \\
1-6 Months & 2 & 5.7 \\
6 Months to 1 year & 4 & 11 \\
1-2 Years & 2 & 5.7 \\
2-3 Years & 3 & 8.6 \\
$>3$ Years & 13 & 37 \\
\hline
\end{tabular}

The end points were survival without recurrent hemorrhage from SCAVMs.

\section{Statistical Analysis}

Statistical analysis was performed by using SPSS for Mac (Version 21.0; IBM, Armonk, New York). Variables are expressed as mean $\pm \mathrm{SD}$, median (interquartile range: 25 th -75 th percentile), or number of patients (percentage) as appropriate. The normality of the data was evaluated by using the Shapiro-Wilk test. We performed receiver operating characteristics curve analysis for age and the binary end point of recurrent hemorrhage, and we selected the age cutoff point that optimized sensitivity and specificity.

We compared the baseline and radiologic characteristics of patients with and without recurrent hemorrhage by the log-rank test and performed multivariate analysis by the Cox proportional hazards model by using variables that were marginally or significantly associated with progression on the log-rank test $(P<.20)$. The probability of freedom from recurrent hemorrhage was estimated by using the Kaplan-Meier method; comparisons of the survival curves by the number of factors were performed by using the log-rank test. Differences were considered significant at $P<$ .05 for a $95 \%$ CI.

\section{RESULTS}

Forty-four patients (55\%) presented with subarachnoid hemorrhage and 36 (45\%) presented with hematomyelia. Types of initial hemorrhage were not related to recurrent hemorrhage $(P=.54)$. We observed recurrent hemorrhage in 35 (44\%) patients, including 1 of 41 patients treated by embolization and 34 of 39 patients not treated by embolization. The median length of total follow-up was 659 days (interquartile range, 129-2640 days), and the median length from the first-to-recurrent hemorrhage was 369 days (interquartile range, 30-1596 days). We found that $31 \%$ of patients rebled within 1 month of the first hemorrhage and $40 \%$ of them rebled in $>3$ years (Table 1 ). Associated diseases were noted in several patients: spinal arterioveonous metameric syndrome in 10 patients (13\%) with recurrent hemorrhage and in $9(20 \%)$ without recurrent hemorrhage; Klippel-Trenaunay-Weber syndrome in $1(2.9 \%)$ with recurrent hemorrhage, not seen in those without recurrent hemorrhage; and Osler-Weber-Rendu disease in $1(2.9 \%)$ with recurrent hemorrhage, not seen in those without recurrent hemorrhage. No significant difference was found among these associated diseases and recurrent hemorrhage $(P=$ $.94)$. Radiation therapy was performed in 3 patients (8.6\%) with recurrent hemorrhage and was not performed in patients without recurrent hemorrhage $(P=.42)$. Clinical and radiologic charac- teristics of 80 patients with and without recurrent hemorrhage are shown in Table 2. The log-rank test revealed that endovascular treatment (Fig 2) and venous thrombosis significantly and marginally reduced recurrent hemorrhage, respectively, and an associated aneurysm was significantly related to recurrent hemorrhage. Even in multivariate analysis, the endovascular treatment reduced and associated aneurysm increased the risk of recurrent hemorrhage (Table 3).

\section{Illustrative Case}

This male patient initially presented with a spinal subarachnoid hemorrhage at 11 years of age. A diagnosis of an SCAVM was made by MR imaging and spinal angiography at that time, but no treatment was performed. He then experienced 3 spinal subarachnoid hemorrhages in a month, documented by spinal tap at 14 years of age. He was then referred to us for endovascular treatment. On admission, he was neurologically intact. Angiographic study demonstrated a nidus-type SCAVM at the T2 spinal level supplied mainly by the anterior spinal artery (ASA) with multiple intranidal aneurysms (Fig 3A). Superselective angiography from a feeder coming off the ASA failed to demonstrate the aneurysms (Fig $3 B,-C$ ). Superselective angiography from the ASA near the feeder origin demonstrated an intranidal aneurysm supplied by a branch of the ASA different from the feeder that was previously catheterized (Fig 3D). Because of the inability to superselectively catheterize the feeder supplying the aneurysm, embolization from the ASA near the origin of the feeder was performed by using NBCA (Fig 3E). Control angiography after the embolization showed decreased size of the AVM without opacification of the aneurysms. The ASA was disconnected for a short segment, but the distal segment was opacified through the collateral. The remaining nidus was also opacified by the ASA and the posterior spinal artery opacified from the left T10 and the left T8 intercostal artery, respectively (not shown). The patient remained neurologically intact and had no further hemorrhage during the follow-up for 6 years after the embolization.

\section{DISCUSSION}

The results of the present study showed that the endovascular treatment reduced and associated aneurysm increased the risk of recurrent hemorrhage in patients with hemorrhagic SCAVM.

\section{Hemorrhage}

The congenital nature of SCAVMs in their form at presentation is also debatable, ${ }^{13}$ because their appearance after hemorrhage may reflect the result of decompensation of the vasculature lesion and the surrounding spinal cord, which occurred after birth. It has previously been reported that once a patient hemorrhages, the incidence of recurrent hemorrhage is very high. ${ }^{14}$ The overall annual hemorrhage rate was $4 \%$, increasing to $10 \%$ for spinal glomus (type 2) SCAVMs with previous hemorrhage, ${ }^{8}$ and lesions with initial hemorrhage also had a greater annual rate of recurrent hemorrhage $(5.6 \%)$ compared with nonhemorrhagic lesions $(0.4 \%)$ in spinal cord AVF. ${ }^{15}$ Our series showed an higher incidence of hemorrhage: $58 \%$ as the initial symptom and $66 \%$ before treatment. In the literature, initial hemorrhage was encountered in $70 \%$ of pediatric and $27 \%-45 \%$ of adult patients with 
Table 2: Univariate freedom from recurrent hemorrhage of SCAVMs ${ }^{\mathrm{a}}$

\begin{tabular}{|c|c|c|c|c|}
\hline \multirow[b]{2}{*}{ Variables } & \multirow[b]{2}{*}{ Total $(n=80)$} & \multicolumn{2}{|c|}{ Recurrent Hemorrhage } & \multirow{2}{*}{$\begin{array}{c}\text { Unadjusted } \\
P \text { Value }\end{array}$} \\
\hline & & $(+)(n=35)$ & $(-)(n=45)$ & \\
\hline \multicolumn{5}{|l|}{ Baseline characteristics } \\
\hline Median age (yr) (IQR) & $20(10-32)$ & $18(8-25)$ & $21(15-38)$ & \\
\hline Pediatric & $30(38)$ & $16(46)$ & $14(31)$ & .87 \\
\hline Male & $31(39)$ & $12(34)$ & $19(42)$ & .73 \\
\hline Endovascular embolization ${ }^{\text {b }}$ & $41(51)$ & $1(2.9)$ & 40 (89) & $<.0001$ \\
\hline \multicolumn{5}{|l|}{ Radiologic characteristics } \\
\hline Subclassification & & & & .51 \\
\hline SCAVM & $63(79)$ & $31(89)$ & $32(71)$ & \\
\hline SCAVF, single hole & $7(8.8)$ & $1(2.9)$ & $6(13)$ & \\
\hline SCAVF, multiple holes & 10 (13) & 4 (11) & $6(13)$ & \\
\hline SCAVM level & & & & .92 \\
\hline Cranial-cervical & $2(2.5)$ & $1(2.9)$ & $1(2.2)$ & \\
\hline Cervical & $33(41)$ & $16(46)$ & $17(39)$ & \\
\hline Cervical-thoracic & $1(1.3)$ & 0 & $1(2.2)$ & \\
\hline Thoracic & $31(39)$ & $13(37)$ & $18(40)$ & \\
\hline Thoracic-lumbar & 9 (11) & 4 (11) & 5 (11) & \\
\hline Lumbar & $4(5.0)$ & $1(2.9)$ & $3(6.7)$ & \\
\hline Associated aneurysm ${ }^{\mathrm{b}}$ & $56(70)$ & $31(89)$ & $25(56)$ & .049 \\
\hline Venous thrombosis ${ }^{b}$ & $7(8.8)$ & $1(2.9)$ & $6(13)$ & .19 \\
\hline Venous stricture & $2(2.5)$ & 0 & $2(4.4)$ & .21 \\
\hline Venous ectasia & $16(20)$ & $7(20)$ & $9(20)$ & .24 \\
\hline Venous hypertension & $16(20)$ & $7(20)$ & $9(20)$ & .66 \\
\hline
\end{tabular}

Note:-SCAVF indicates spinal cord AVF; IQR, interquartile range 25th-75th percentile.

${ }^{a}$ Data are expressed as number of lesions (\%), unless otherwise indicated.

${ }^{\mathrm{b}}$ Variables related to the recurrent hemorrhage by log-rank test $(P<.20)$.

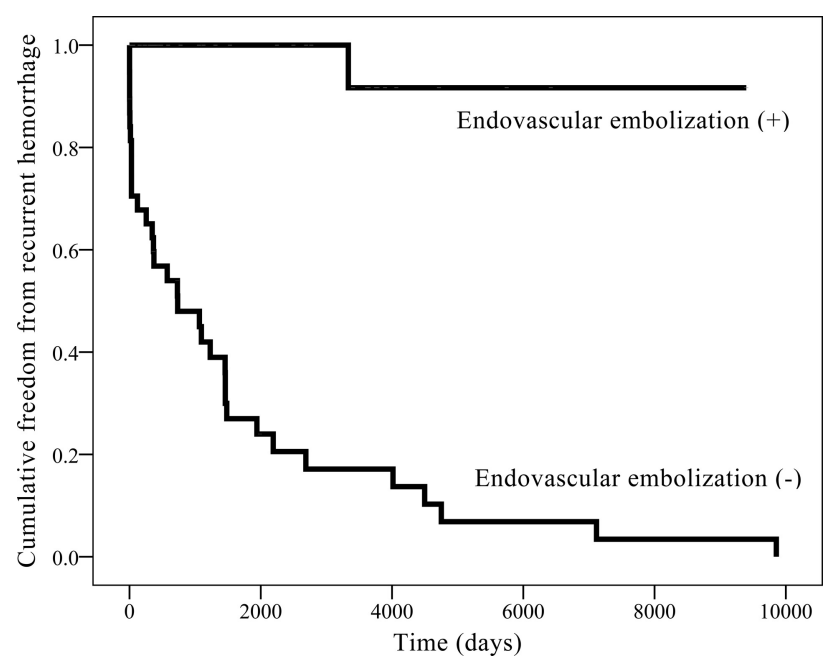

FIG 2. Kaplan-Meier plot of cumulative freedom from the progression by endovascular embolization.

Table 3: Multivariate analyses using the Cox proportional hazards model for recurrent hemorrhage from SCAVMs

\begin{tabular}{lccc}
\hline \multicolumn{1}{c}{ Variable } & $\begin{array}{c}\text { Adjusted } \\
\text { HR }\end{array}$ & $\mathbf{9 5 \% ~ C l}$ & $\begin{array}{c}\text { Multivariable } \\
\text { Adjusted } \\
\text { P Value }\end{array}$ \\
\hline Endovascular treatment $^{\mathrm{a}}$ & 0.027 & $0.0040-0.19$ & $<.0001$ \\
Associated aneurysm $^{\mathrm{a}}$ & 3.4 & $1.2-11$ & .044 \\
Venous thrombosis & 0.61 & $0.082-4.5$ & .63 \\
\hline
\end{tabular}

Note:- - HR indicates hazard ratio.

${ }^{a}$ Variables related to recurrent hemorrhage.

SCAVMs, $20 \%-37 \%$ of pediatric and adult patients with AVF, and $50 \%$ of pediatric and adult patients with glomus-type AVMs. ${ }^{6,8,9,15,16}$ In the present study, initial hemorrhage was seen in $52 \%$ of all patients, $51 \%$ of pediatric patients, and $53 \%$ of adults, and this finding supported the results of the previous studies. Previous study also showed that early (within the 12 months after the first hemorrhage) recurrent hemorrhage was seen in $4.8 \%$ of pediatric patients and in $3.6 \%$ of adults, and late $(>1$ year after the first hemorrhage) recurrent hemorrhage was seen in $4.8 \%$ of pediatric patients and $14 \%$ of adults with SCAVM. ${ }^{6}$ However, early recurrent hemorrhage was seen in $20 \%$ of pediatric patients and in $20 \%$ of adults, and late recurrent hemorrhage was seen in $33 \%$ of pediatric patients and in $18 \%$ of adults in the present study. This result indicated that the rate of recurrent hemorrhage from SCAVMs in both pediatric patients and adults was higher than previously expected.

Recurrent hemorrhages after endovascular embolization were seen in $4 \%$, and all of these were cervical SCAVMs in the previous study. ${ }^{17}$ We observed that only 1 of 41 patients (2.4\%) had recurrent hemorrhage among those who underwent endovascular treatment, as opposed to 34 of 39 patients $(87.2 \%)$ without endovascular treatment. Many of those who had rehemorrhage without endovascular treatment were treated by endovascular embolization without further hemorrhage in our series (data not shown). The low incidence of hemorrhage after partial embolization of SCAVMs has also been reported by other authors. ${ }^{5}$

Associated aneurysms of the feeding arteries and nidus are common in SCAVMs. ${ }^{7}$ Spinal cord artery and intranidal aneurysms are associated with a high risk for hemorrhage in SCAVMs. ${ }^{7}$ Some studies have shown that SCAVM-related arterial aneurysms (distinct from false aneurysms) were associated with initial hemorrhage, ${ }^{6,8,14,18}$ though one group of authors showed that arterial aneurysms (distinct from false aneurysms) were not related to hemorrhagic presentation. ${ }^{16}$ It was also reported that recurrent hemorrhages were mostly due to rerupture of an associated false aneurysm in SCAVMs. ${ }^{6}$ The results of the present study showed that associated aneurysms were seen in $89 \%$ of patients with recurrent hemorrhage and in $56 \%$ of those without recurrent hemorrhage and supported the relationship between associated aneurysms and recurrent hemorrhage in patients with an SCAVM.

Although venous architectural factors (either venous ectasias or venous stricture) were most often associated with initial hemorrhage, ${ }^{16}$ they were not related to recurrent hemorrhage in the present study.

\section{Endovascular Treatment}

Embolization of an SCAVM is performed once the endovascular "dissection" of the SCAVM with microcatheter angiography and provocative testing is complete. Analysis of vascular anatomy is most important for safe embolization, but electrophysiologic 

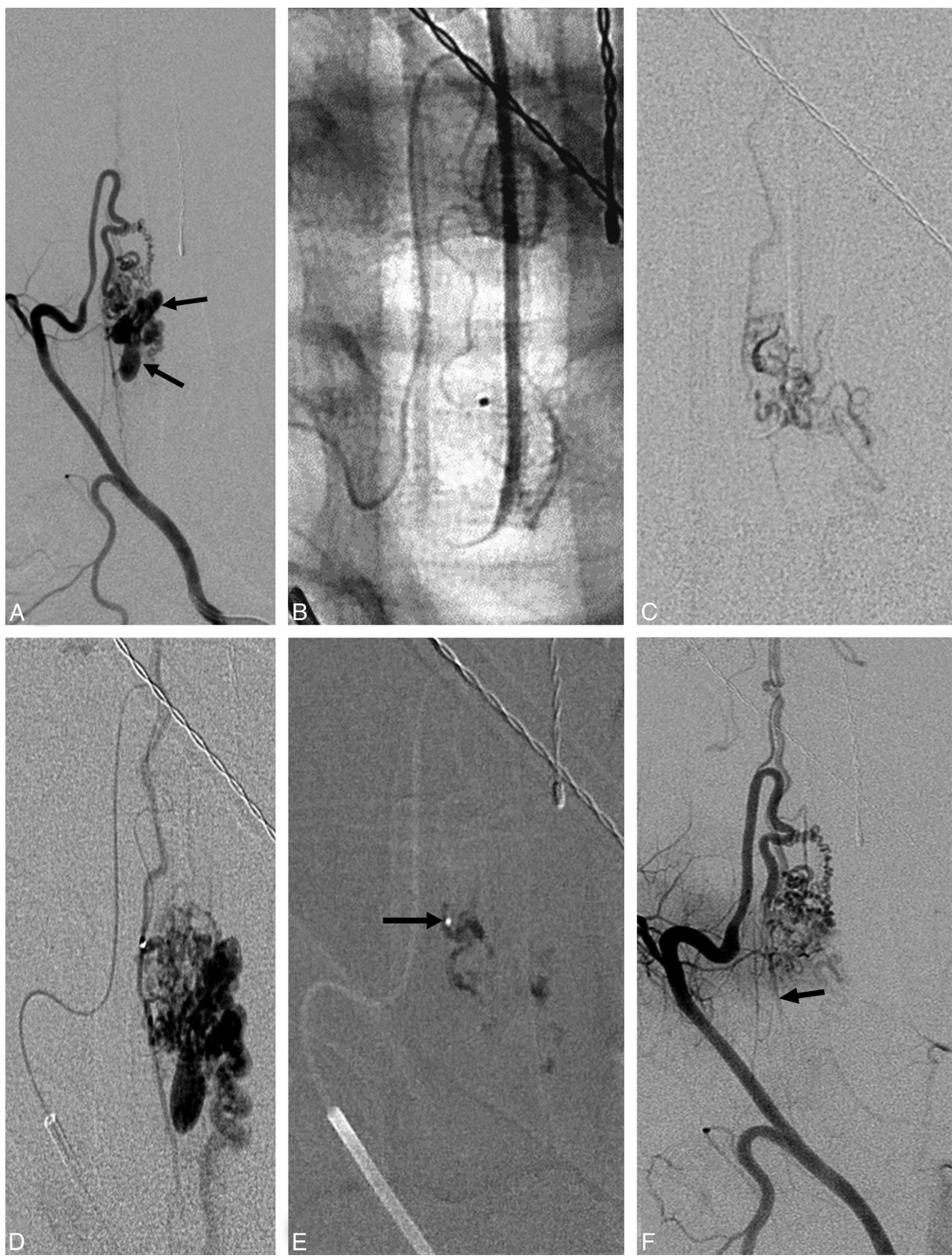

FIG 3. A, Posteroanterior view of the right $\mathrm{T} 2$ intercostal artery angiogram showing a nidus-type SCAVM supplied by the anterior spinal artery with multiple intranidal aneurysms (arrows). B, Nonsubtracted image of the microcatheter inserted into a feeder to the SCAVM. C, Superselective angiogram from the microcatheter inserted into the same feeder as $B$. No aneurysms are visualized. $D$, Superselective angiogram from a microcatheter placed in the ASA at the origin of the feeder to the SCAVM. Intranidal aneurysms and the distal anterior spinal artery are visualized. Embolization was performed from this catheter position using NBCA. E, Cast of NBCA. A small amount of NBCA is in the ASA axis near the catheter tip (arrow). F, Posteroanterior view of the right T2 intercostal artery after embolization. There is decreased visualization of the nidus without opacification of the intranidal aneurysms. The distal ASA is seen through the collateral (arrow). 
monitoring with provocative testing provides additional safety, especially when vascular anatomy is modified by previous hemorrhage or treatment. ${ }^{12}$ We prefer to use a flow-guided microcatheter for embolization because it is smaller in caliber and less traumatic to the blood vessel compared with a guidewire-assisted microcatheter. We prefer to use NBCA as an embolic agent because of its penetration small vessels and long-lasting occlusion effect. We try to reach the target as close as possible, but if not possible, we modify our technique to accomplish occlusion of the target as demonstrated in the illustrative case. We avoid embolization from a microcatheter wedged in a feeding artery because of the high risk of penetration of the embolic material into the normal territory or draining veins. The details of embolization techniques have been described elsewhere. ${ }^{12,14}$ We proved that targeted embolization is beneficial to prevent recurrent hemorrhage even if it is partial treatment. We believe that endovascular embolization is the treatment of choice for most SCAVMs. The only exception is slow-flow, perimedullary AVFs located at the conus or filum terminale. It is important to obtain periodic clinical and radiologic follow-up and early endovascular intervention before significant deterioration occurs to obtain the best possible prognosis for SCAVMs.

\section{Limitations}

First, our study had a retrospective design with inherent limitations leading to potential ascertainment bias. Second, the number of patients was small, spread across many years, during which technical advancements were observed, though this may be unavoidable given that SCAVM is a rare disease. Third is the possibility of random error; overall, 13 univariate comparisons have been performed. Given the $\alpha$ level of .10, the high number of statistical tests makes it likely that 2 of the associations found may actually be the result of chance. Therefore, the interpretation of the results of univariate analysis was performed carefully, and the conclusion was performed by the multivariate analysis. As noted above, the results of the present study should be further clarified using an independent cohort would be difficult to perform given the rarity of SCAVMs.

\section{CONCLUSIONS}

The low rate of recurrent hemorrhage after endovascular treatment, compared with the high incidence of recurrent hemorrhage in conservative management, proves that endovascular treatment is effective to prevent hemorrhage.

\section{REFERENCES}

1. Veznedaroglu E, Nelson PK, Jabbour PM, et al. Endovascular treatment of spinal cord arteriovenous malformations. Neurosurgery 2006;59(5 suppl 3):S202-09; discussion S3-13 Medline

2. Kim LJ, Spetzler RF. Classification and surgical management of spinal arteriovenous lesions: arteriovenous fistulae and arteriovenous malformations. Neurosurgery 2006;59:S195-201; discussion S3-13 CrossRef Medline
3. Zozulya YP, Slin'ko EI, Al-Qashqish II. Spinal arteriovenous malformations: new classification and surgical treatment. Neurosurg Focus 2006;20:E7 Medline

4. Inagawa S, Yamashita S, Hiramatsu $\mathrm{H}$, et al. Clinical results after the multidisciplinary treatment of spinal arteriovenous fistulas. Jpn J Radiol 2013;31:455-64 CrossRef Medline

5. Biondi A, Merland JJ, Reizine D, et al. Embolization with particles in thoracic intramedullary arteriovenous malformations: long-term angiographic and clinical results. Radiology 1990;177:651-58 CrossRef Medline

6. Rodesch G, Hurth M, Alvarez H, et al. Angio-architecture of spinal cord arteriovenous shunts at presentation: clinical correlations in adults and children. Acta Neurochir (Wien) 2004;146:217-26; discussion 226-27 CrossRef Medline

7. Biondi A, Merland JJ, Hodes JE, et al. Aneurysms of spinal arteries associated with intramedullary arteriovenous malformations, II: results of AVM endovascular treatment and hemodynamic considerations. AJNR Am J Neuroradiol 1992;13:923-31 Medline

8. Gross BA, Du R. Spinal glomus (type II) arteriovenous malformations: a pooled analysis of hemorrhage risk and results of intervention. Neurosurgery 2013;72:25-32; discussion 32 CrossRef Medline

9. Mourier KL, Gobin YP, George B, et al. Intradural perimedullary arteriovenous fistulae: results of surgical and endovascular treatment in a series of 35 cases. Neurosurgery 1993;32:885-91; discussion 891 Medline

10. Rosenblum B, Oldfield EH, Doppman JL, et al. Spinal arteriovenous malformations: a comparison of dural arteriovenous fistulas and intradural AVM's in 81 patients. J Neurosurg 1987;67:795-802 CrossRef Medline

11. von Elm E, Altman DG, Egger M, et al; STROBE Initiative. The Strengthening the Reporting of Observational Studies in Epidemiology (STROBE) statement: guidelines for reporting observational studies. Lancet 2007;370:1453-57 CrossRef Medline

12. Niimi Y, Sala F, Deletis V, et al. Neurophysiologic monitoring and pharmacologic provocative testing for embolization of spinal cord arteriovenous malformations. AJNR Am J Neuroradiol 2004;25: 1131-38 Medline

13. Lasjaunias P. A revised concept of the congenital nature of cerebral arteriovenous malformations. Interv Neuroradiol 1997;3:275-81 CrossRef Medline

14. Berenstein A, Lasjaunias P, TerBrugge K. Spinal arterioenous malformations. In: Surgical Neuroangiography. Berlin Heidelberg: Springer-Verlag; 2004:737-847

15. Gross BA, Du R. Spinal pial (type IV) arteriovenous fistulae: a systematic pooled analysis of demographics, hemorrhage risk, and treatment results. Neurosurgery 2013;73:141-51; discussion 151 CrossRef Medline

16. Rodesch G, Hurth M, Alvarez H, et al. Spinal cord intradural arteriovenous fistulae: anatomic, clinical, and therapeutic considerations in a series of 32 consecutive patients seen between 1981 and 2000 with emphasis on endovascular therapy. Neurosurgery 2005; 57:973-83; discussion 973-83 CrossRef Medline

17. Rodesch G, Hurth M, Alvarez H, et al. Embolization of spinal cord arteriovenous shunts: morphological and clinical follow-up and results_-review of 69 consecutive cases. Neurosurgery 2003;53:40 -49; discussion 49-50 CrossRef Medline

18. Konan AV, Raymond J, Roy D. Transarterial embolization of aneurysms associated with spinal cord arteriovenous malformations: report of four cases. J Neurosurg 1999;90(1 suppl):148-54 CrossRef Medline 